\title{
ARTICLE OPEN Enhanced superconductivity in TiO epitaxial thin films
}

\author{
Chao Zhang ${ }^{1}$, Feixiang $\mathrm{Hao}^{1}$, Guanyin Gao ${ }^{1}$, Xiang $\mathrm{Liu}^{1}$, Chao Ma ${ }^{1}$, Yue Lin ${ }^{1}$, Yuewei Yin ${ }^{1,2}$ and Xiaoguang $\mathrm{Li}^{1,3,4}$
}

Titanium oxides have many fascinating optical and electrical properties, such as the superconductivity at $2 \mathrm{~K}$ in cubic titanium monoxide (TiO) polycrystalline bulk. However, the lack of TiO single crystals or epitaxial films has prevented systematic investigations on its superconductivity. Here, we report the basic superconductivity characterizations of cubic TiO films epitaxially grown on (0001)-oriented $\mathrm{a}-\mathrm{Al}_{2} \mathrm{O}_{3}$ substrates. The magnetic and electronic transport measurements confirmed that TiO is a type-II superconductor and the recorded high $T_{\mathrm{c}}$ is about $7.4 \mathrm{~K}$. The lower critical field $\left(H_{\mathrm{c} 1}\right)$ at $1.9 \mathrm{~K}$, the extrapolated upper critical field $H_{\mathrm{c} 2}(0)$, and coherence length are about $18 \mathrm{Oe}, 13.7 \mathrm{~T}$, and $4.9 \mathrm{~nm}$, respectively. With increasing pressure, the value of $T_{\mathrm{c}}$ shifts to lower temperature while the normal state resistivity increases. Our results on the superconducting TiO films confirm the strategy to achieve higher $T_{\mathrm{c}}$ in the epitaxial films, which may be helpful for finding more superconducting materials in various related systems.

npj Quantum Materials (2017)2:2 ; doi:10.1038/s41535-016-0006-3

\section{INTRODUCTION}

Transition metal oxides, such as titanium oxides, are a large family of materials with many fascinating electrical properties and applications. ${ }^{1-3}$ Among various stable titanium oxides, the cubic metallic monoxide $\mathrm{TiO}$ is one of the very interesting materials because of the extremely wide homogeneity range, with oxygen content varying from about 0.80 to $1.30 .^{4-7}$ The electrical, optical, magnetic, and structural properties of bulk TiO have been widely investigated. ${ }^{8-17}$ Especially, the superconductivity in TiO bulk materials was discovered in the early 1965 s by Hulm et al. ${ }^{18}$ They reported that, for the bulk $\mathrm{TiO}$ with $\mathrm{NaCl}$ structure, its superconducting transition temperature $T_{\mathrm{c}}$ increases from 0.2 to $1.0 \mathrm{~K}$ with oxygen content increases from 0.9 to 1.1 , but is below $0.08 \mathrm{~K}$ outside this range. ${ }^{19}$ Further investigations showed that highpressure annealing increases the oxygen content and lattice constant of cubic TiO bulk, and its superconducting transition temperature increases linearly with oxygen content to a maximum of $2.0 \mathrm{~K}^{4,7}$ However, the intrinsic superconducting properties of $\mathrm{TiO}$, such as the lower and upper critical fields, superconducting coherence length and so on, are not clear yet, due to the difficulty in obtaining single crystals or epitaxial films. Even the zero resistance superconducting state has not been clearly reported. Therefore, high-quality TiO epitaxial thin films or single crystals are essential and highly desired for investigating the fundamental superconductivity of the system. There was only one $\sim 10 \mathrm{~nm}$ TiO single crystalline film reported very recently, which was formed on titanium dioxide $\left(\mathrm{TiO}_{2}\right)$ substrate through the surface chemical reduction method by a low-energy ion bombardment technique. ${ }^{20}$ Although this method is good at creating a thin TiO film on $\mathrm{TiO}_{2}$ substrate, no magnetic and electrical properties concerning the superconductivity were reported. In fact, it is still a challenge to prepare high-quality TiO epitaxial films on different substrates through a more controllable method like magnetron sputtering or pulsed laser deposition techniques.
On the other hand, it is well known that, for epitaxial superconducting thin films, the superconductivity could be enhanced or even created by proper heterostructure interfaces, such as the enhanced $T_{\mathrm{c}}$ above $100 \mathrm{~K}$ in the epitaxial FeSe films grown on $\mathrm{SrTiO}_{3}$ substrates, which was explained in terms of the coupling between conduction electrons and the substrate phonons, ${ }^{21-23}$ as well as the superconductivity created at the interface between two insulators like $\mathrm{LaAlO}_{3} / \mathrm{SrTiO}_{3}{ }^{24,25}$ In addition, a suitable lattice mismatch-induced strain can also enhance the $T_{\mathrm{c}}$ as reported in $\mathrm{La}_{1.9} \mathrm{Sr}_{0.1} \mathrm{CuO}_{4}{ }^{26}$ Therefore, it will be interesting to see whether the superconductivity in TiO films can be improved as well.

In the present work, we successfully prepared cubic TiO thin films on a (0001)-oriented a- $\mathrm{Al}_{2} \mathrm{O}_{3}$ single crystal substrate by a pulsed laser deposition technique. The superconducting transition temperature of $7.4 \mathrm{~K}$ was observed for the TiO films, confirmed by magnetization and electrical transport measurements. The $T_{\mathrm{c}}$ is almost four times higher than its bulk value and is suppressed with increasing pressure (maximum pressure $1.8 \mathrm{GPa}$ ).

\section{RESULTS AND DISCUSSION}

$\mathrm{TiO}$ thin films with the thickness of $\sim 80 \mathrm{~nm}$ were epitaxially grown on (0001)-oriented $\mathrm{a}-\mathrm{Al}_{2} \mathrm{O}_{3}$ single crystalline substrates (see Methods). The structural characterization of the TiO thin films was performed using high-angle annular dark-field-scanning transmission electron microscope (HAADF-STEM) and X-ray diffraction (XRD). It was determined that the cubic TiO thin film grown on $\mathrm{a}-\mathrm{Al}_{2} \mathrm{O}_{3}(0001)$ substrate is of the [111] direction perpendicular to its surface. Figure 1 a shows the cross-sectional HAADF-STEM image at the TiO/a- $\mathrm{Al}_{2} \mathrm{O}_{3}$ interface region of an asgrown sample viewed along the [1100] direction of sapphire, and thus the formation of a highly epitaxial TiO film is confirmed with a 2-3 atomic transition layer at the interface. The epitaxial relationship between the cubic TiO layer and sapphire substrate

\footnotetext{
${ }^{1}$ Hefei National Laboratory for Physical Sciences at the Microscale, Department of Physics, University of Science and Technology of China, Hefei 230026 , China; ${ }^{2}$ Department of

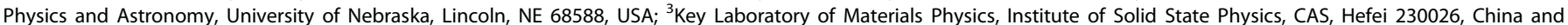
${ }^{4}$ Collaborative Innovation Center of Advanced Microstructures, Nanjing 210093, China Correspondence: Yuewei Yin (yyin11@unl.edu) or Xiaoguang Li (lixg@ustc.edu.cn)
}

Received: 5 August 2016 Revised: 5 November 2016 Accepted: 22 November 2016 Published online: 20 January 2017 


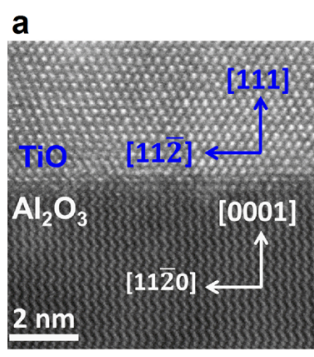

\section{b}

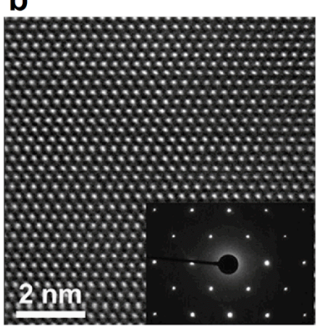

C

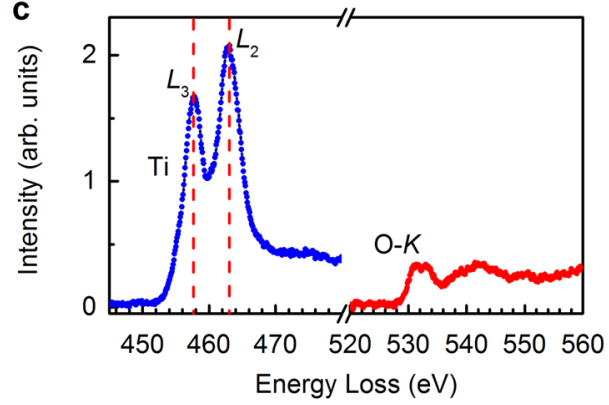

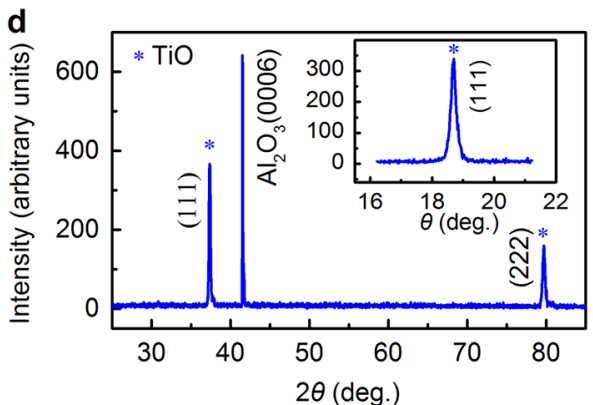

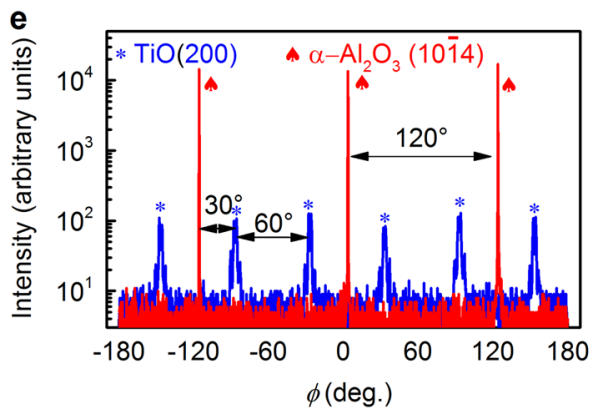

Fig. 1 Structural and chemical characterizations. a,b HAADF-STEM images of $\mathrm{TiO} / \mathrm{Al}_{2} \mathrm{O}_{3}$, at the a interface region and $\mathbf{b}$ TiO region. $\mathbf{c}$ Ti- $L_{3,2}$ and O-K edges EELS spectra of the TiO film. $\mathbf{d} \theta / 2 \theta$ XRD pattern of the TiO/ $\alpha-\mathrm{Al}_{2} \mathrm{O}_{3}(0001)$ heterostructure. The inset shows the rocking curve for the TiO (111) reflection. e XRD $\phi$ scan performed on TiO (200) and sapphire (10 $\overline{1} 4)$ planes

is determined as $\mathrm{TiO}[11 \overline{2}](111) / / \mathrm{a}-\mathrm{Al}_{2} \mathrm{O}_{3}[11 \overline{2} 0](0001)$. Figure $1 \mathrm{~b}$ shows the HAADF-STEM image and the related electron diffraction pattern in the TiO region viewed along the [1T00] direction of sapphire as well, which shows single crystalline quality and agrees well with the simulation using the face-centered cubic TiO structure (see Supplementary Information Fig. S1). Chemical and oxidation state analyses of the TiO film were performed by the electron energy-loss spectroscopy (EELS) in STEM, as shown in Fig. 1c. For titanium oxides, the fine structures of the Ti- $L_{3,2}$ and $\mathrm{O}-K$ edges reflect the covalent bonding states resulting from strong hybridization between Ti-3d and $\mathrm{O}-2 p$ electronic states. For our sample, the Ti- $L_{3,2}$ and $O-K$ edges both consist mainly of two peaks, which represent the fingerprint feature of cubic TiO (space group $F m \overline{3} m) .{ }^{27}$ The EELS spectra recorded from different areas of the TiO film indicate the structure homogeneity in the obtained TiO film, as shown in Supplementary Information Fig. S2. Through the quantitative analysis of EELS results, ${ }^{28}$ it was found that, as shown in Fig. S2, the oxygen content in the film is not uniform and the averaged $\mathrm{O} / \mathrm{Ti}$ ratio is about $1.11-1.25$, which is similar to that reported by Pabon et al. ${ }^{20}$

Although the combination of STEM images with electron diffraction pattern is a powerful tool in determining the crystal structure of materials, the results provide only local structural information of a small volume. Therefore, we carried out detailed XRD experiments. Figure $1 d$ shows the XRD $\theta / 2 \theta$ specular scan of an as-grown $\mathrm{TiO}$ thin film on $\mathrm{a}-\mathrm{Al}_{2} \mathrm{O}_{3}$ (0001) substrate. Only the (111) family diffractions of TiO film and the sapphire (0006) diffraction peak were observed, indicating that the film is highly orientated in [111] direction parallel to sapphire [0001]. The cubic lattice constant (about $4.164 \AA$ ) of the TiO film is calculated from the peak position of the (111) plane using Bragg's law, which is very close to the TiO bulk value of $4.177 \AA$. One of the reasons for the difference in lattice parameters between the TiO film and the bulk may be related to the oxygen content, because it was reported that the lattice parameter could be reduced with increasing oxygen content., 19,20 As shown in the inset of Fig. 1d, the full width at half maximum of the TiO (111) plane rocking curve is about $0.3^{\circ}$, indicating its good crystallinity. To obtain the in-plane epitaxial relationship between the TiO film and sapphire substrate more clearly, XRD $\phi$-scans of the (200) plane of the TiO film and the (1014) plane of $\mathrm{a}-\mathrm{Al}_{2} \mathrm{O}_{3}$ substrate were performed, as shown in Fig. 1e. For the TiO film, the six distinct peaks at $60^{\circ}$ intervals to each other with nearly the same intensity indicate a six-fold rotational symmetry along the TiO (111) plane normal. From the XRD $\phi$-scan, an epitaxial relation as TiO $[11 \overline{2}](111) / / a-\mathrm{Al}_{2} \mathrm{O}_{3}$ [11 20$](0001)$ was obtained, consistent with the STEM results shown in Figs. $1 a, b$.

The existence of the superconducting phase in the TiO film was unambiguously confirmed by its Meissner effect and zero resistance. Figure 2a shows the direct-current (DC) magnetizations vs. temperature $(M-T)$ of the TiO film. Both the field-cooled (FC) and zero-field-cooled (ZFC) magnetizations in 20 Oe magnetic field perpendicular to the film surface indicate the appearance of superconductivity near $7.0 \mathrm{~K}$, much higher than that of $\mathrm{TiO}$ polycrystalline bulk $(0.2 \sim 2.0 \mathrm{~K}){ }^{4,19}$ The irreversible region of magnetizations marked by the bifurcation of FC and ZFC curves below $T_{\mathrm{c}}$ shows smaller FC signal as compared with the ZFC signal, indicating the flux trapping by thin film defects during the FC process. The magnetization vs. magnetic field curve $M-H$ at $1.9 \mathrm{~K}$ for a magnetic field perpendicular to the film surface $(H \perp(111))$ is shown in Fig. 2b. The lower critical field $H_{c 1}$, defined as the field at which a flux first penetrates, can be estimated from the $M-H$ curve as a deviation from the linear $M-H$ behavior corresponding to the Meissner state. The evaluated value is about $18 \mathrm{Oe}$ at $1.9 \mathrm{~K}$ for $H \perp(111)$ as shown in the inset of Fig. $2 b$.

The superconducting property of the TiO film was also investigated through electrical transport measurements, using the Hall bar pattern. The schematic diagram of the experimental setup is shown in Supplementary Information Fig. S3. Figure 3 shows the temperature-dependent resistance $\left(R_{x x}\right)$ in zero magnetic field and Hall resistance $\left(R_{x y}\right)$ in 2 T magnetic field. In the $R_{x x}-T$ curve, the resistance rises with decreasing temperature first, and a kink $\left(T_{\text {kink }}\right)$ appears around $130 \mathrm{~K}$, below which the resistance increases even steeper. This kink may be related to the charge localization. ${ }^{29,30}$ When the temperature goes down further, the resistance decreases suddenly and the superconducting behavior occurs at $\sim 7.4 \mathrm{~K}$, as shown in the inset of Fig. 3a. The 
a

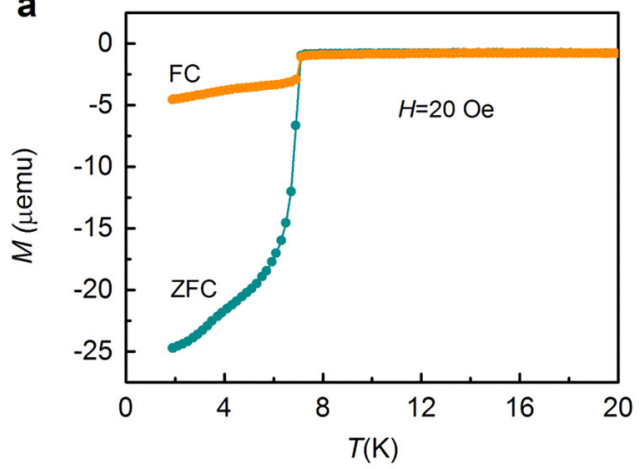

b

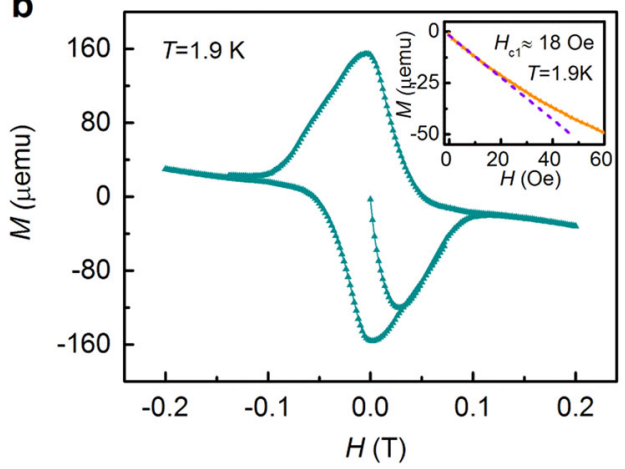

Fig. 2 Magnetization characterizations. a Temperature dependence of the DC magnetization of TiO film in ZFC and FC modes at $20 \mathrm{Oe}$ for $H \perp(111)$. The $T_{c}$ is identified as $7.0 \mathrm{~K}$. b Magnetization vs. magnetic field $(M-H)$ at $1.9 \mathrm{~K}$ for $H \perp(111)$. Inset: $H_{\mathrm{c} 1}$ at $1.9 \mathrm{~K}$ was estimated to be about $18 \mathrm{Oe}$

superconducting resistive transition at zero field is relatively broad, which may be attributed to the following two aspects: (i) inhomogeneous oxygen or titanium stoichiometry of the films, as shown in Fig. S2, and (ii) a consequence of a Berezinskii-Kosterlitz-Thouless (BKT)-like transition, as described in the following equation: ${ }^{31}$

$R_{x y}(T)=R_{\mathrm{N}} \exp \left[-b\left(\frac{T-T_{\mathrm{BKT}}}{T_{\mathrm{BKT}}}\right)^{(-1 / 2)}\right]$,

where $R_{N}$ is normal-state sheet resistance, and $b$ is a nonuniversal dimensionless numerical constant. The good fitting result (blue solid line in inset of Fig. 3(a)) indicates that the resistive transition may be a consequence of the BKT-like transition $\left(T_{\mathrm{BKT}}=4.91 \mathrm{~K}\right)$ probably related to the $\mathrm{TiO}$ film- $\mathrm{Al}_{2} \mathrm{O}_{3}$ substrate 2D interface, which localizes and enhances the superconductivity through conduction electron-substrate phonon interaction, similar to that occurred in FeSe thin films. ${ }^{22,23}$

From the Hall result, it is confirmed that the $n$-type electronic charge carriers dominate the conduction mechanism, as shown in Fig. 3b. According to the relation $R_{\mathrm{H}}=R_{x y} d / H=1 / n q$, where $R_{\mathrm{H}}, d, n$, and $q$ are the Hall coefficient, film thickness, charge carrier density, and carrier charge, respectively, the carrier concentration was estimated to be about $2.0 \times 10^{22} \mathrm{~cm}^{-3}$ at $300 \mathrm{~K}$. It is noted that the Hall voltage near the superconducting transition temperature has a sign opposite to the voltage in the normal state, as shown in the inset of Fig. 3b, which may be related to the unusual vortex motion. ${ }^{32,33}$

Figures $4 a$, $b$ show the temperature-dependent resistances with different magnetic fields parallel $(H / /(111))$ and perpendicular $(H \perp(111))$ to the TiO film surface. The parallel resistance broadenings in different fields are clearly observed, and the superconducting transition shifts to lower temperatures with increasing magnetic field. Figure $4 \mathrm{c}$ shows the upper critical field $H_{\mathrm{c} 2}(T)$ and irreversibility field $H_{\text {irr }}(T)$ of the TiO film in the in-plane and out-of-

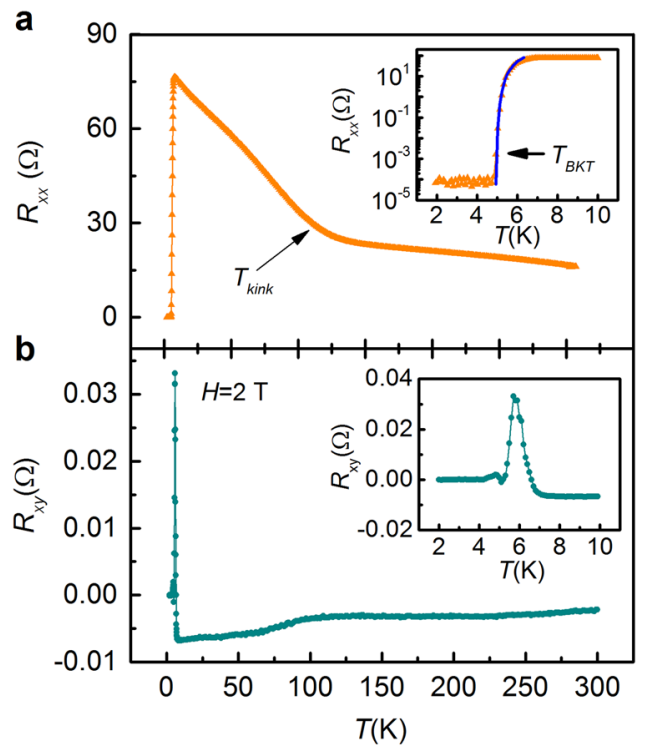

Fig. 3 Electrical transport measurements. a Temperature dependence of the resistance $R_{x x}$ from 1.9 to $300 \mathrm{~K}$. b Temperature dependence of Hall resistance $R_{x y}$ at a magnetic field of $2 \mathrm{~T}$. The insets are the corresponding magnified figures around $T_{\mathrm{c}}$ and the blue solid line is the fitting result by BKT-like transition

plane configurations, determined using the criterions of $90 \%$ and $0.1 \%$ normal-state resistance (see Supplementary Fig. S4 for the detailed information). ${ }^{34,35}$ The upper critical field vs. temperature curves can be well fitted by: ${ }^{36}$

$H_{\mathrm{c} 2}(T)=H_{\mathrm{c} 2}(0)\left[1-\left(\frac{T}{T_{\mathrm{c}}}\right)^{2}\right]$

where $H_{\mathrm{c} 2}(0)$ is the upper critical field at absolute zero temperature. The extrapolated values of $H_{\mathrm{c} 2}$ are about 13.6 and 13.7 T for magnetic fields perpendicular $(H \perp(111))$ and parallel $(H / /$ (111)) to the TiO film surface, respectively. Correspondingly, the Landau-Ginzburg superconducting coherence lengths, $\xi=[(h / 2 e) /$ $\left.\left(2 \pi H_{\mathrm{c} 2}\right)\right]^{1 / 2}$, at absolute zero temperature are estimated to be about 4.92 and $4.91 \mathrm{~nm}$ for $H \perp(111)$ and $H / /(111)$, respectively. According to the anisotropic effective mass Ginzburg-Landau theory, ${ }^{37}$ the anisotropy ratio $\varepsilon \sim 1.01$ was obtained from the scaling law $\varepsilon=\left(m_{\perp} / m_{/ /}\right)^{0.5}=H_{c 2}^{/ /} / H_{c 2}^{\perp}$. Here $H_{c 2}^{\perp}$ and $H_{c 2}^{/ /}$are the upper critical fields for $H \perp(111)$ and $H / /(111)$, and $m_{\perp}$ and $m_{/ /}$are the effective masses of electrons along these directions, respectively. As for the temperature dependence of $H_{\text {irr, }}$ it can be well fitted by: ${ }^{36}$

$H_{\text {irr }}(T)=H_{\text {irr }}(0)\left(\frac{T_{c}-T}{T_{c}}\right)^{n}$

with $n=0.85$. To investigate the anisotropic superconducting properties in more detail, we studied the magnetic field orientation dependence of the superconducting transition. Figure $4 \mathrm{~d}$ shows the field dependences of the resistances at different angles $\beta$ at $4.0 \mathrm{~K}$, where $\beta$ denotes the tilt angle between the normal of film plane and the field direction, as depicted in the inset of Fig. 4 d. One can see that $H_{\mathrm{c} 2}$ changes a little, while $H_{\text {irr }}$ increases gradually with increasing $\beta$ from $H \perp(111)$ to $H / /(111)$.

Figure 5 shows the pressure effect on the superconducting transition of the TiO film. With increasing pressure, the zeroresistance transition temperature shifts to lower temperatures and the normal-state resistivity increases obviously. It may be due to the pressure-enhanced charge localization, and the effective attraction of the Cooper pairs is thus suppressed as a result.

From the above results and discussions, it is clear that a significant $T_{\mathrm{c}}$ enhancement was observed in the TiO epitaxial thin film. Although the underlying physics mechanism is not well 

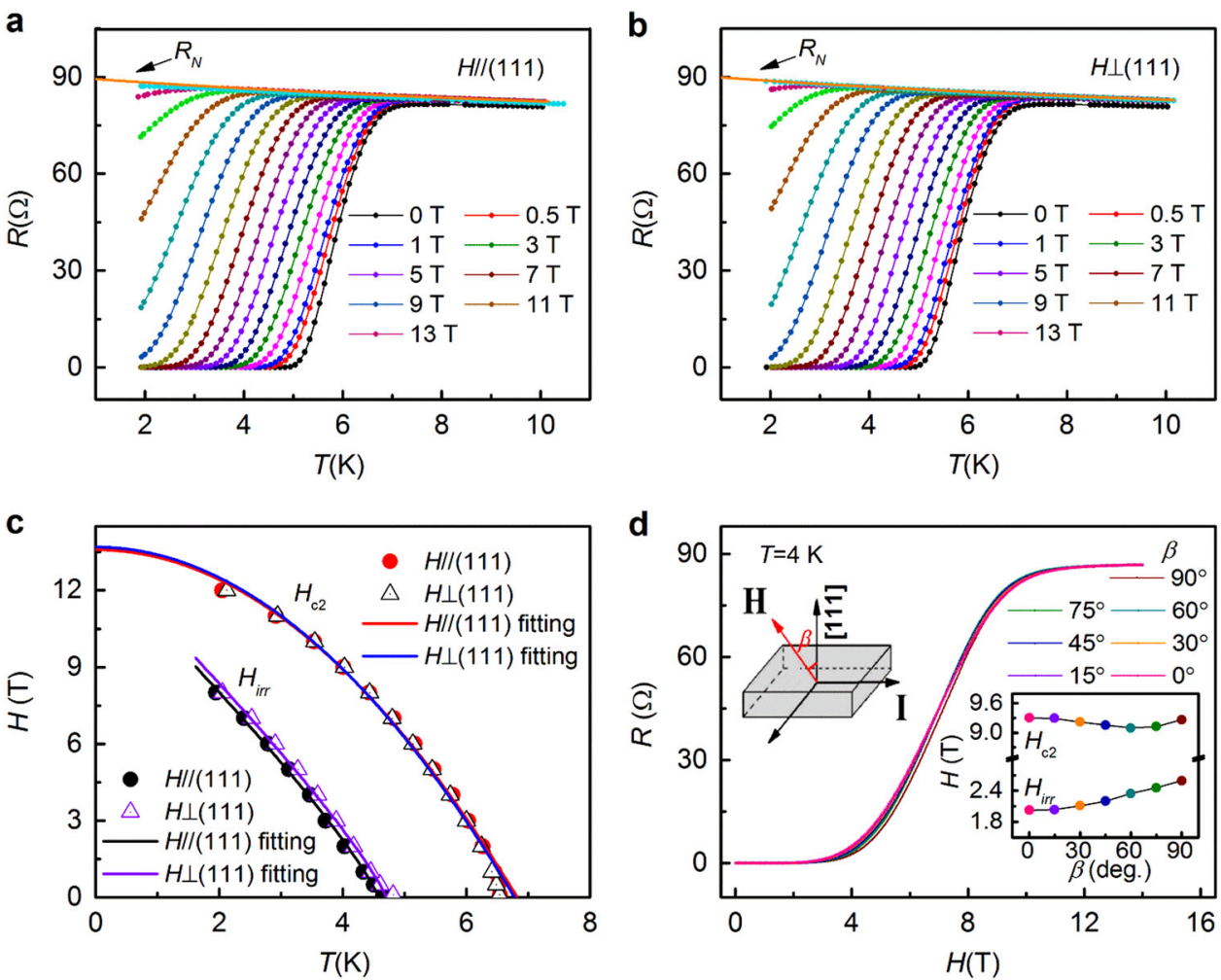

Fig. 4 Upper critical field and irreversibility field. $\mathbf{a}, \mathbf{b}$ Temperature-dependent resistances in different magnetic fields a parallel $(H / /(111))$ and $\mathbf{b}$ perpendicular $(H \perp(111))$ to the TiO film surface. c Temperature-dependent $H_{\mathrm{c} 2}$ and $H_{\text {irr }}$ in different magnetic field directions $H \perp(111)$ and $H / /$ (111). The hollow triangles and solid dots are experimental results deduced from (a) and (b), and the lines are the fitting results. $\mathbf{d}$ Magnetic field dependencies of the resistances at $4.0 \mathrm{~K}$ with different angles $\beta$ (defined as the inset). The inset shows magnetic field angle dependences of $H_{\text {irr }}$ and $H_{\mathrm{c} 2}$

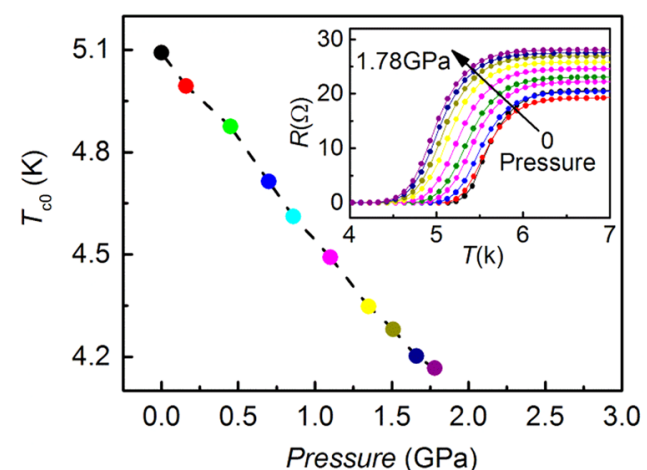

Fig. 5 Pressure dependence of zero-resistance transition temperature. Inset: $R_{x x}-T$ curves at different pressures

understood, our results suggest several clues for the occurrence of superconductivity. First, inhomogeneous oxygen or titanium stoichiometry was detected from the detailed STEM analysis, which may significantly affect the local electronic structure as well as the superconductivity in the cubic TiO film. Second, the BKT-like transition in the $\mathrm{TiO}$ thin films, similar to the FeSe thin films, ${ }^{23}$ may be probably related to the $\mathrm{TiO}-\mathrm{Al}_{2} \mathrm{O}_{3}$ interface through the conduction electron-substrate phonon interaction. ${ }^{22}$ Third, the high-pressure experiments demonstrate the importance of strain effect on the superconductivity. The strain status in the TiO film can be influenced by not only the substrate strain but also the oxygen stoichiometry. Further experimental and theoretical investigations on the micro-stoichiometry, structure, and electronic properties both in the non-uniform films and at the $\mathrm{TiO}-\mathrm{Al}_{2} \mathrm{O}_{3}$ interface are required to finalize the mechanism of the enhanced $T_{\mathrm{c}}$.
In conclusion, the superconducting cubic TiO thin films were epitaxially grown on the (0001)-oriented $\mathrm{a}-\mathrm{Al}_{2} \mathrm{O}_{3}$ single crystalline substrates, and the superconducting properties of the films were systematically characterized. It was found that the superconducting transition temperature is enhanced to about $7.4 \mathrm{~K}$, and the upper critical field at zero field $H_{\mathrm{c} 2}(0)$ is about $13.7 \mathrm{~T}(H / /(111))$ in the $\mathrm{TiO}$ thin films. The high-pressure experimental results indicated that increasing pressure weakens the superconductivity. Our work will facilitate prospects for understanding the superconducting mechanism in the titanium-based oxide superconductors as well as achieving higher temperature superconductivity.

\section{METHODS}

Sample preparation

The TiO thin films with the thickness of about $80 \mathrm{~nm}$ were epitaxially grown on commercial (0001)-oriented $\mathrm{a}-\mathrm{Al}_{2} \mathrm{O}_{3}$ single crystalline substrates by a pulsed laser deposition technique. Prior to the film deposition, the substrates were annealed in flowing oxygen at $1100^{\circ} \mathrm{C}$ for $120 \mathrm{~min}$. A KrF excimer laser $(\lambda=248 \mathrm{~nm})$ was employed to ablate the $\mathrm{Ti}_{2} \mathrm{O}_{3}$ target. The chamber was evacuated to a base pressure of $\sim 1 \times 10^{-7}$ Torr and purged three times with high-purity nitrogen gas. Then, the films were deposited at $900^{\circ} \mathrm{C}$ and cooled down to room temperature naturally in high vacuum. The laser energy density, repetition rate, and target-substrate distance used for the deposition were $3.5-4.0 \mathrm{~J} \mathrm{~cm}^{-2}, 5 \mathrm{~Hz}$, and $4.5 \mathrm{~cm}$, respectively.

\section{Structural characterizations}

High-resolution XRD measurements were performed with a commercial Panalytical X'pert X-ray diffractometer with the $\mathrm{Cu} \mathrm{Ka}{ }_{1}$ radiation at a wavelength of $1.5406 \AA$. For the structural and chemical characterization of the TiO/Al ${ }_{2} \mathrm{O}_{3}$ samples, a JEOL JEM-ARM200F operating at $200 \mathrm{kV}$, equipped with a spherical aberration corrector on the condenser lens system, was used to obtain the HAADF STEM images and core-level EELS spectra. The thickness and surface roughness of the TiO films were measured by STEM 
and atomic force microscope scanning probe microscope (AFM MultiMode V), as shown in Supplementary Information Fig. S5.

\section{Hall bar fabrication}

Ohmic contact electrodes of gold/titanium films with a Hall bar geometry were fabricated by an ultraviolet light-engraving machine JKG-2A and ion beam-sputtering coating system DPS-LIM, which enabled measurements of the four-terminal resistance and Hall coefficient of the channel. A schematic illustration is shown in Supplementary Information Fig. S3. The dimensions of the channel were $500 \mu \mathrm{m}$ in width and $3 \mathrm{~mm}$ in length. The resistance and the Hall coefficient were measured in a Physical Property Measurement System (PPMS-14, Quantum Design).

\section{Electrical and magnetic measurements}

Magnetization measurements were carried out using a Squid Vibrating Sample Magnetometer (Quantum Design). The angle-resolved in-plane fourprobe resistance measurements were performed for the TiO films rotated from out-of-plane to in-plane of the film in magnetic fields up to $14 \mathrm{~T}$ in a PPMS-14 (Quantum Design). The angle $\beta=0^{\circ}$ is defined as the applied magnetic field that was perpendicular to the surface of the TiO film $(H \perp(111))$.

\section{High-pressure experiments}

The pressure dependences of resistances of the TiO film were measured in a pressure cell of PPMS-9 (Quantum Design). The value of the pressure was demarcated by the $T_{\mathrm{c}}$ of $\mathrm{Sn}$ metal $\left(T_{\mathrm{c}}=3.72 \mathrm{~K}\right.$ at atmospheric pressure for $\left.\mathrm{Sn}\right)$.

\section{ACKNOWLEDGEMENTS}

This work was supported by the Natural Science Foundation of China (51332007, 21521001, and 51622209) and by the National Basic Research Program of China (2015CB921201, 2016YFA0300103, and 2012CB922003).

\section{AUTHOR CONTRIBUTIONS}

X.G.L. and Y.W.Y. designed and supervised the experiments. C.Z. and H.F.X. fabricated the sample and performed electric transport, SEM and AFM measurements. G.Y.G. carried out XRD measurements. X.L. and C.Z. performed electronic transport measurements under high pressure. C.M., Y.L. and C.Z. carried out STEM-HAADF and EELS measurements and analyzed the relevant data; X.G.L., C.Z. and H.F.X. were responsible for all data analysis and wrote the manuscript. All the authors contributed to the discussions and editing of the manuscript.

\section{COMPETING INTERESTS}

The authors declare no competing interests.

\section{REFERENCES}

1. Jeong, D. S. et al. Emerging memories: resistive switching mechanisms and current status. Rep. Prog. Phys. 75, 076502 (2012).

2. Yang, J. J., Strukov, D. B. \& Stewart, D. R. Memristive devices for computing. Nat. Nanotechnol 8, 13-24 (2013).

3. Jin, K. et al. Anomalous magnetoresistance in the spinel superconductor $\mathrm{LiTi}_{2} \mathrm{O}_{4}$. Nat. Commun. 6, 7183 (2015).

4. Reed, T. B., Banus, M. D., Sjöstrand, M. \& Keesom, P. H. Superconductivity in cubic and monoclinic "TiO". J. Appl. Phys. 43, 2478-2479 (1972).

5. Martev, I. N. Oxygen-ion-assisted deposition of TiO films. Vacuum 58, 327-334 (2000)

6. Watanabe, D. \& Castles, J. R. The ordered structure of TiO. Acta Crystallogr 23, 307-313 (1967)

7. Banus, M. D. Quenchable effects of high pressures and temperatures on the cubic monoxide of titanium. Mater. Res. Bull. 3, 723-734 (1968).

8. Xu, W.-X., Zhu, S., Fu, X.-C. \& Chen, Q. The structure of $\mathrm{TiO}_{x}$ thin film studied by Raman spectroscopy and XRD. Appl. Surf. Sci. 148, 253-262 (1999).

9. He, Q. et al. Thermoelectric property studies on bulk $\mathrm{TiO}_{x}$ with $x$ from 1 to 2 . Appl. Phys. Lett. 91, 052505 (2007).

10. Valeeva, A. A., Rempel, A. A. \& Gusev, A. I. Electrical conductivity and magnetic susceptibility of titanium monoxide. JETP Lett 73, 621-625 (2001).

11. Sreemany, M., Bose, A. \& Sen, S. A study on structural, optical, electrical and microstructural properties of thin $\mathrm{TiO}_{x}$ films upon thermal oxidation: Effect of substrate temperature and oxidation temperature. Physica B 405, 85-93 (2010).
12. Valeeva, A. A., Nazarova, S. Z. \& Rempel, A. A. In situ study of atomic-vacancy ordering in stoichiometric titanium monoxide by the magnetic susceptibility. JETP Lett 101, 258-263 (2015).

13. Bartkowski, S. \& Neumann, M. Electronic structure of titanium monoxide. Phys. Lett. B 56, 10656-10667 (1996).

14. Doyle, N. et al. Vacancies and superconductivity in titanium monoxide. Phys. Lett. A 26, 604-605 (1968).

15. Banakh, O., Schmid, P. E., Sanjines, R. \& Levy, F. Electrical and optical properties of $\mathrm{TiO}_{x}$ thin films deposited by reactive magnetron sputtering. Surf. Coat. Technol 151, 272-275 (2002)

16. Yao, J., Shao, J., He, H. \& Fan, Z. Optical and electrical properties of $\mathrm{TiO}_{x}$ thin films deposited by electron beam evaporation. Vacuum 81, 1023-1028 (2007).

17. Bally, A. R. et al. Mechanical and electrical properties of fcc $\mathrm{TiO}_{1+x}$ thin films prepared by r.f. reactive sputtering. Surf. Coat. Technol 108-109, 166-170 (1998).

18. Daunt, J. G., Edwards, D. O., Milford, F. J. \& Yaqub, M. in IXth International Conference on Low Temperature Physics Vol. 2 (ed. Hulm, J. K. et al.) Ch. 5.5 (Plenum Press, 1965).

19. Hulm, J. K., Jones, C. K., Hein, R. A. \& Gibson, J. W. Superconductivity in the TiO and NbO Systems. J. Low Temp. Phys. 7, 291-307 (1972).

20. Pabon, B. M. et al. Formation of titanium monoxide (001) single-crystalline thin film induced by ion bombardment of titanium dioxide (110). Nat. Commun. 6 6147 (2015).

21. Ge, J. F. et al. Superconductivity above $100 \mathrm{~K}$ in single-layer FeSe films on doped $\mathrm{SrTiO}_{3}$. Nat. Mater. 14, 285-289 (2015)

22. Li, Z. X., Wang, F., Yao, H. \& Lee, D. H. What makes the $T_{c}$ of monolayer FeSe on $\mathrm{SrTiO}_{3}$ so high: a sign-problem-free quantum Monte Carlo study. Sci. Bull 61, 925-930 (2016).

23. Liu, D. et al. Electronic origin of high-temperature superconductivity in singlelayer FeSe superconductor. Nat. Commun. 3, 931 (2012).

24. Reyren, N. et al. Superconducting interfaces between insulating oxides. Science 317, 1196-1199 (2007)

25. Mohanta, N. \& Taraphder, A. Multiband theory of superconductivity at the $\mathrm{LaAlO}_{3} / \mathrm{SrTiO}_{3}$ interface. Phys. Lett. B 92, 174531 (2015).

26. Locquet, J.-P. et al. Doubling thecritical temperature of $\mathrm{La}_{1.9} \mathrm{Sr}_{0.1} \mathrm{CuO}_{4}$ using epitaxial strain. Nature 394, 453-456 (1998).

27. Stoyanov, E., Langenhorst, F. \& Steinle-Neumann, G. The effect of valence state and site geometry on $\mathrm{Ti}_{3,2}$ and $\mathrm{O} \mathrm{K}$ electron energy-loss spectra of $\mathrm{Ti}_{\mathrm{x}} \mathrm{O}_{\mathrm{y}}$ phases. Am. Mineral. 92, 577-586 (2007).

28. Egerton, R. F. Electron energy-loss spectroscopy in the electron microscope (Springer Science \& Business Media, 2011).

29. Marezio, M., McWhan, D. B., Dernier, P. D. \& Remeika, J. P. Charge localization at metal-insulator transitions in $\mathrm{Ti}_{4} \mathrm{O}_{7}$ and $\mathrm{V}_{4} \mathrm{O}_{7}$. Phys. Rev. Lett. 28, 1390-1393 (1972).

30. Kowalski, P. M. et al. Charge localization dynamics induced by oxygen vacancies on the $\mathrm{TiO}_{2}$ (110) surface. Phys. Rev. Lett. 105, 146405 (2010).

31. Kosterlitz, J. M. \& Thouless, D. J. Ordering, metastability and phase transitions in two-dimensional systems. J. Phys. C: Solid State Phys 6, 1181-1203 (1973).

32. Zechner, G. et al. Sign change of the vortex hall effect in superconducting YBCO thin films with a square pattern of ion-irradiated defect columns. Physica $C$ (http://dx.doi.org/10.1016/j.physc.2016.06.001, in press).

33. Hagen, S. J., Lobb, C. J., Greene, R. L. \& Eddy, M. Flux-flow hall effect in superconducting $\mathrm{Tl}_{2} \mathrm{Ba}_{2} \mathrm{CaCu}_{2} \mathrm{O}_{8}$ films. Phys. Lett. B 43, 6246-6248 (1991).

34. Lei, $\mathrm{H}$. et al. Iron chalcogenide superconductors at high magnetic fields. Sci. Technol. Adv. Mat. 13, 054305 (2012).

35. Alexandrov, A. S., Zavaritsky, V. N., Liang, W. Y. \& Nevsky, P. L. Resistive upper critical field of high- $T_{c}$ single crystals of $\mathrm{Bi}_{2} \mathrm{Sr}_{2} \mathrm{CaCu}_{2} \mathrm{O}_{8}$. Phys. Rev. Lett. 76 983-986 (1996).

36. Mazov, L. S. Symmetry and heterogeneity in high temperature superconductors. (Springer, the Netherlands, 2006).

37. Blatter, G., Geshkenbein, V. B. \& Larkin, A. I. From isotropic to anisotropic superconductors: A scaling approach. Phys. Rev. Lett. 68, 875-878 (1992).

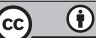

This work is licensed under a Creative Commons Attribution 4.0 International License. The images or other third party material in this article are included in the article's Creative Commons license, unless indicated otherwise in the credit line; if the material is not included under the Creative Commons license, users will need to obtain permission from the license holder to reproduce the material. To view a copy of this license, visit http://creativecommons.org/licenses/by/ $4.0 /$

(c) The Author(s) 2017

Supplementary Information accompanies the paper on the npj Quantum Materials website (doi:10.1038/s41535-016-0006-3). 